\title{
Rejection Of Direct Blast Interference Based on Vector Sensor adaptive Array Processing
}

\author{
Yunfei Chen ${ }^{1,2, a}$, Bing Jia ${ }^{2, b}$, Zhenshan Wang ${ }^{2}$, Feng Gao ${ }^{2}$, \\ Sheng $\mathrm{Li}^{1}$, Guijuan $\mathrm{Li}^{2}$ \\ ${ }^{1}$ Department of Ship Engineering, Dalian University of Technology, Dalian, 116024, China \\ ${ }^{2}$ Science and Technology on Underwater Test and Control Laboratory, Dalian, 116013, China \\ aemail: yunfeidlut@163.com, bemail:jiabing.robin@gmail.com
}

Keywords: Direct Blast Interference Rejection; Vector Sensor; Adaptive Array Processing;

\begin{abstract}
In bistatic acoustic scattering wave testing, there will be strong direct blast interference , An algorithm based on vector sensor adaptive signal processing that rejecting of direct blast interference in bistatic sonar has been studied, through computer simulation the effectiveness of this algorithm was demonstrated. Simulation test results show that when the space angle of interference and signal greater than $5 \mathrm{deg}$, the object scattering signal can be effectively estimated with six elements of vector sensor array
\end{abstract}

\section{Introduction}

In recent years, bistatic or multistatic acoustic scattering testing becomes an effective way of object acoustic characteristics study, because the characteristics of bistatic or multistatic target scattering wave provide more information about the target than the characteristics of monostatic target acoustic scattering wave [1-3], but the testing of bistaic acoustic scattering has the problem of direct blast interference [4][5]. Fig.1 is diagrammatic sketch of direct blast interference in bistatic acoustic testing. In bistatic acoustic testing configuration, the transmitter and the receiver are separated at a certain distance, the transmission loss of direct blast signal is smaller than the signal scattered by underwater object for the direct blast signal transmits directly from transmitter to receiver. When direct blast and object scattering signals arrive at the receiver at the same time, the object scattering signal is impossible to be extracted from the strong direct blast interference.

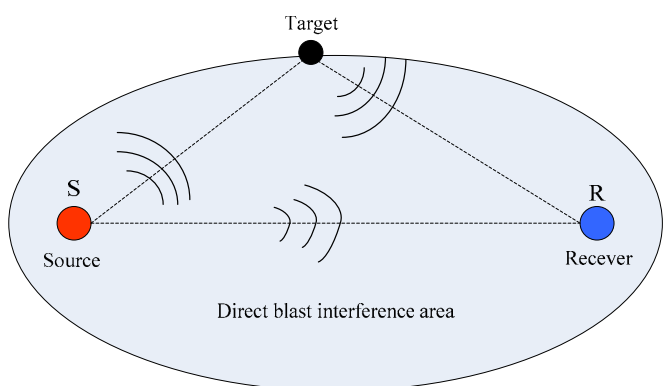

Fig.1. Direct blast interference in bistatic acoustic testing

Recently, the problem of direct blast rejection in bistatic acoustic testing has gained great interest of study. Normally, we use conventional beamforming to separate two signals that arriving from different directions, but this method fails under the condition that interference is much stronger than the signal. Adaptive filtering is another way of interference rejection [4][5][6], through the adaptive null steering processor, the direct blast interference could be suppressed. The performance of this method depends greatly on the beamwidth of conventional beamforming, when the space angle of interference and signal smaller than one beamwidth, namely the object scattering signal and the direct blast interference are in the same receiving beam, adaptive filtering cannot reject the interference accurately.

Many studies have shown that acoustic vector sensor could provide higher directional gain 
compared with hydrophone [7], so the vector sensor array could provide higher directional gain against reverberation with small aperture. In this paper, an algorithm based on vector sensor signal processing and adaptive signal processing has been studied, this algorithm fully integrated the benefits of adaptive filtering and vector sensor array signal processing, which could reject the direct blast interference effectively with small array aperture.

\section{Principle of Direct Blast Rejection based on Vector Sensor adaptive Array Processing}

The principal of adaptive array processing is steering the lowered or null response of a receiving beam pattern in the direction of interference, the width of the null depends on the array beamwidth, when space angle of interference and signal smaller than one beamwidth but not in the same direction, adaptive array processing could reject the interference but also decreased the power of the desired signal. Vector sensor array could provide higher directional gain against reverberation with small aperture, based on that, we can use the adaptive array processing to suppress the direct blast interference and use the vector sensor array processing to increase the gain of the signal to reverberation.

Fig. 2 is the block diagram of adaptive filtering vector sensor array processing rejecting direct blast interference, in which, $\mathrm{CBF}$ means conventional beamforming. The vector sensor array element space is $d$ in bistatic testing. The acoustic vector sensor measure the acoustic pressure and velocity of the scattering wave, there are three channel in one acoustic vector. $\beta$ is the DOA of direct blast interference, which can be accurately obtained through surface positioning system. The DOA of object scattering signal and direct blast are different, the signal received by receiver array is expressed as $S_{i t}(t)$, the signal processing flow of direct blast rejection algorithm is:

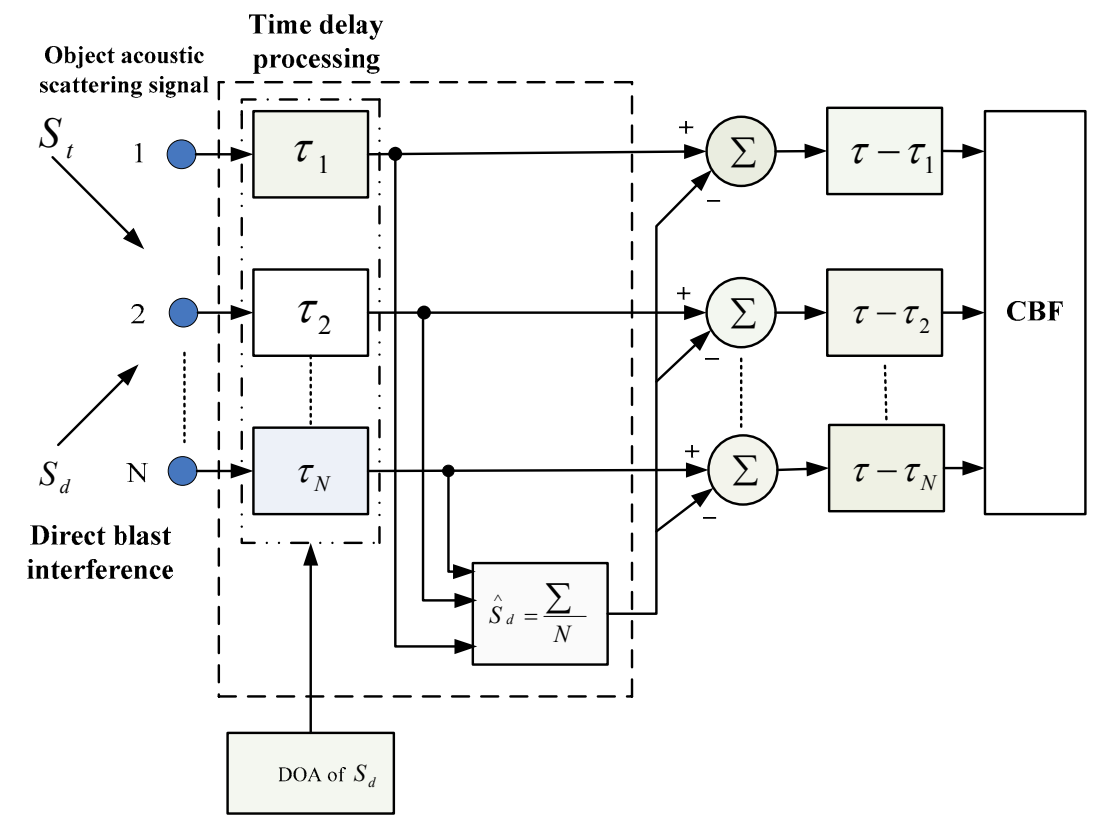

Fig.2. Block diagram of adaptive filtering array processing rejecting direct blast interference

1) Obtaining $\beta$ through shipborne surface positioning system.

2) Time delay processing for hydrophone array received signal, time delay parameter is

$\tau_{d}=\frac{(N-1) d \sin \beta}{c}$

3) Ensemble average for the direct blast signal.

$\hat{S}_{d}=\sum_{N}^{i=1} S_{i}\left(t-\tau_{d}\right) / N$

4) Direct blast rejection for each receiver channel:

$S_{i t}=S_{i}\left(t-\tau_{d}\right)-\widehat{S}_{d}$ 
5) Time delay compensation for each receiver channel after direct blast rejection, time delay parameter is

$$
\tau_{d}=-\frac{(N-1) d \sin \beta}{c}
$$

6) Conventional beamforming for the object scattering signal.

After the adaptive filtering array processing, we can get the object signal but with lower ratio of signal to reverberation. Next procedure, the ratio of signal to reverberation will be increased through vector sensor array processing. Figure 3 is the signal processing flow of vector sensor array signal processing. It should be noted that $v_{c}$ is the synthesized vector value, $p$ is acoustic scattering wave pressure, $v_{x}$ and $v_{y}$ are the velocity of acoustic scattering wave in two orthogonal direction. Acoustic vector sensor could measure acoustic scattering wave pressure and velocity in the same time. The pressure and velocity signal are expressed as:

$$
\begin{aligned}
& p(t)=x(t) \\
& v_{x}(t)=x(t) \cos \theta \\
& v_{y}(t)=x(t) \sin \theta
\end{aligned}
$$

The synthesized vector value $v_{c}$ is expressed as:

$$
v_{c}(t)=v_{x} \cos \theta_{0}+v_{y} \sin \theta_{0}
$$

In which $x(t)$ is the wave form of acoustic pressure and acoustic velocity, the vector array beamforming output signal is:

$$
w=\sum_{i=1}^{N}\left(p+v_{c}\right) \cdot v_{c}
$$

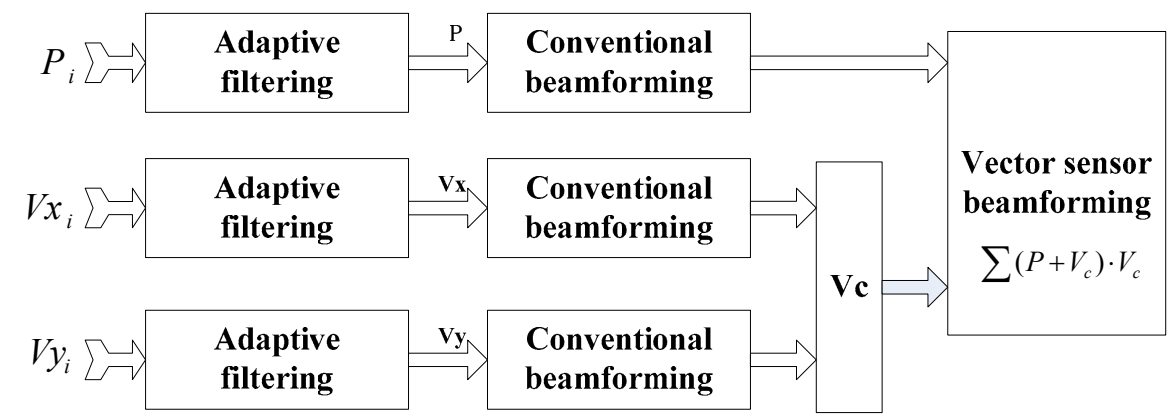

Fig.3. Block diagram of vector sensor adaptive array processing rejecting direct blast interference

\section{Test results}

The effectiveness of the method has been studied through simulation. The simulation parameters are six elements acoustic vector sensors receiving array, element spacing is $0.36 \mathrm{~m}$, source signal is $20 \mathrm{~ms}$ length continuous wave with the frequency of $3000 \mathrm{~Hz}$; Background noise is random white noise, the ratio of signal to noise are $5 \mathrm{~dB}$; The ratio of direct blast interference to object scattering signal is $20 \mathrm{~dB}$; The angle of direct blast interference is 30 degree, and the angle of object scattering wave signal are 45 degree, 40degree, 35 degree, and 31 degree respectively. The beamwidth of conventional beamforming is 17 degree for six elements array; interference and object scattering signal are in the same beam, which satisfied the problem we studied in this paper.

Fig. 4 is the simulated object scattering signal, fig. 5 is the simulated vector sensor received signal combined with noise, direct blast signal and object scattering signal. According to the algorithm above, direct blast rejection is conducted. Fig. 6 is the object scattering signal after direct blast rejection with 15 deg space angle of interference and signal, Fig.7 is the partial enlarged detail of Fig.6. Fig.7 to Fig.10 are the comparative results of the object scattering signal obtained after interference rejection with different space angle of interference and signal, the solid line and the dotted line represent extracted object scattering signal and original signal respectively. 
The results show that when the space angle of interference and signal is greater than 5 deg, the object scattering signal can be effectively estimated. Fig. 11 is the performance comparison of direct blast rejection with different beamwidth, It shows that we can get better performance of direct blast interference rejection with much narrower beamwidth. Summing up the above, the algorithm of vector sensor adaptive array processing can reject the direct blast interference effectively.

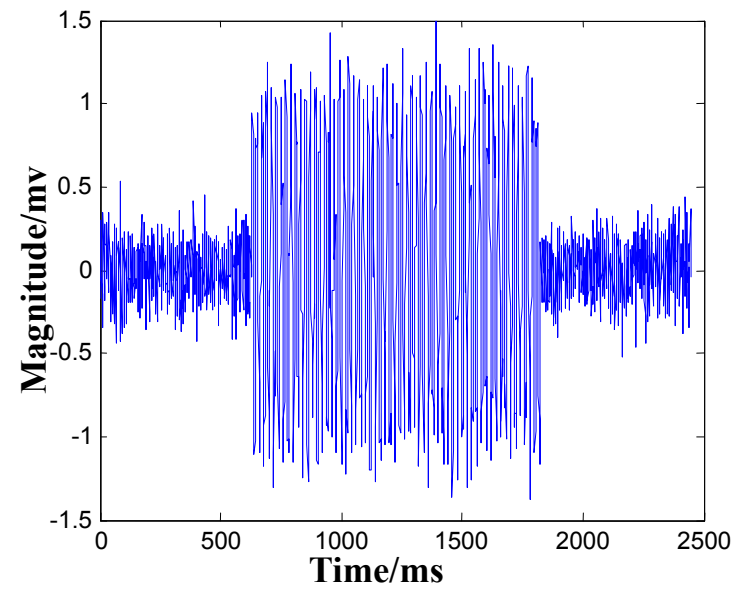

Fig.4. Simulated object scattering signal

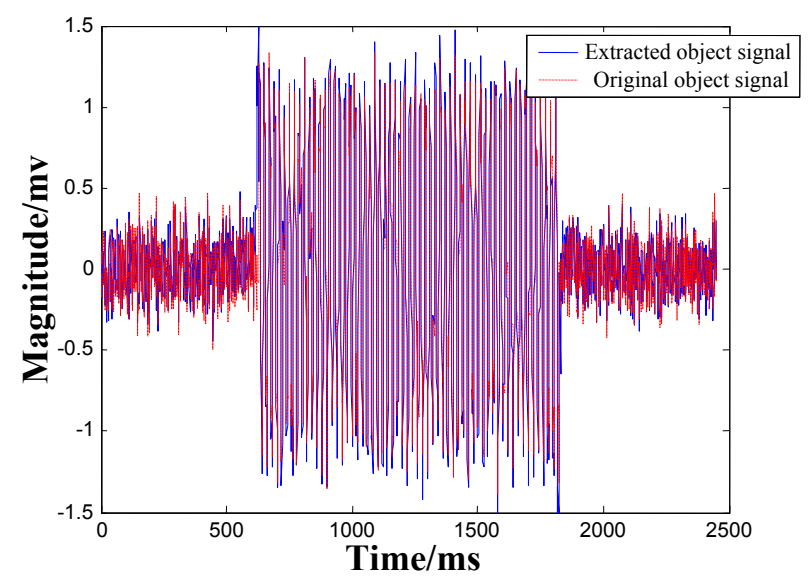

Fig.6. signal after direct blast rejection (space angle of interference and signal is $15 \mathrm{deg}$ )

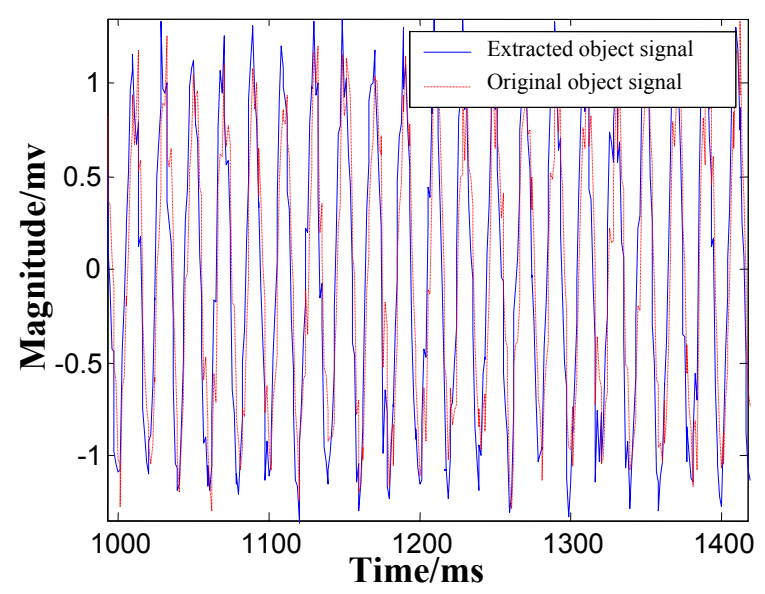

Fig.8. Signal after direct blast rejection (space angle of interference and signal is $10 \mathrm{deg}$ )

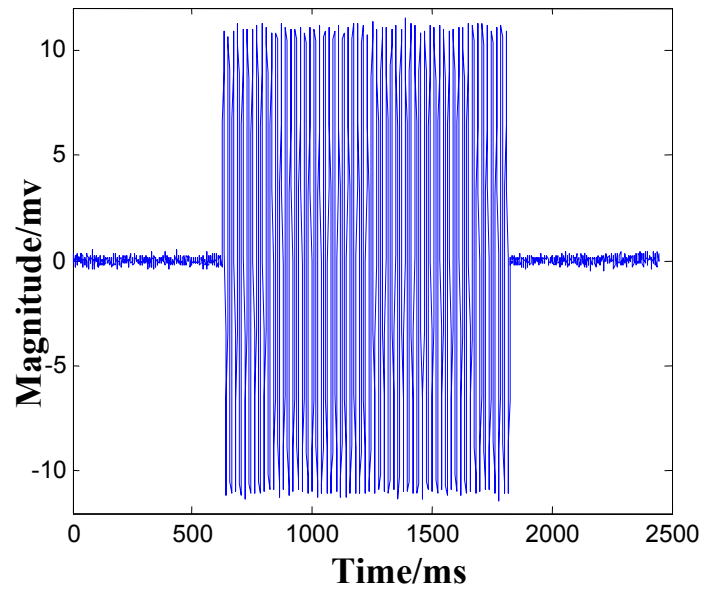

Fig.5. Simulated signal containing with noise, direct blast signal and object scattering signal

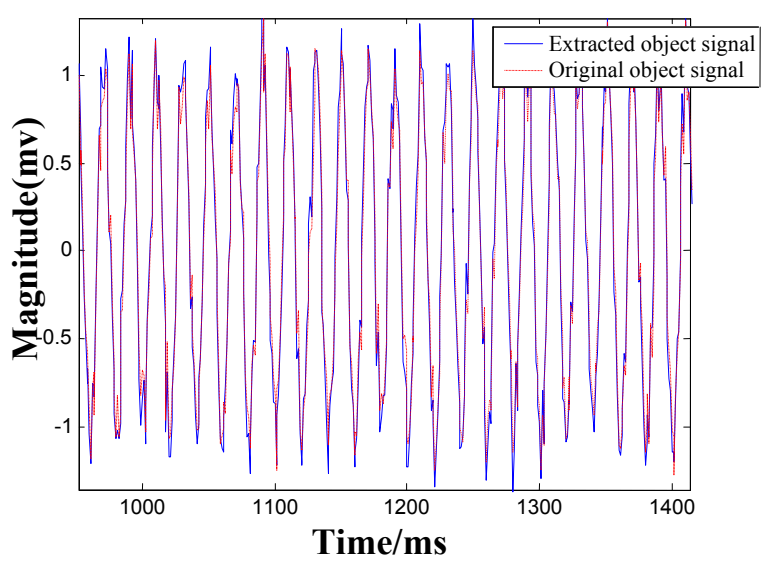

Fig.7. signal after direct blast rejection (space angle of interference and signal is $15 \mathrm{deg}$ )

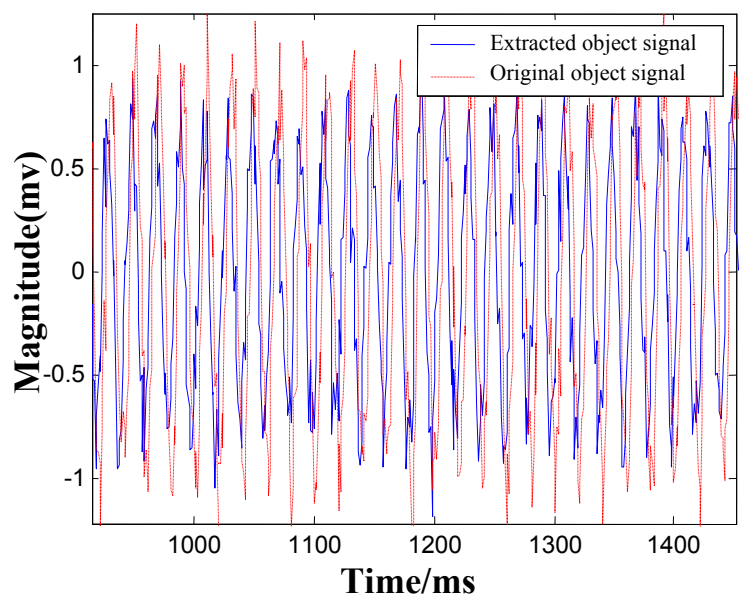

Fig.9. Signal after direct blast rejection (space angle of interference and signal is $5 \mathrm{deg}$ ) 


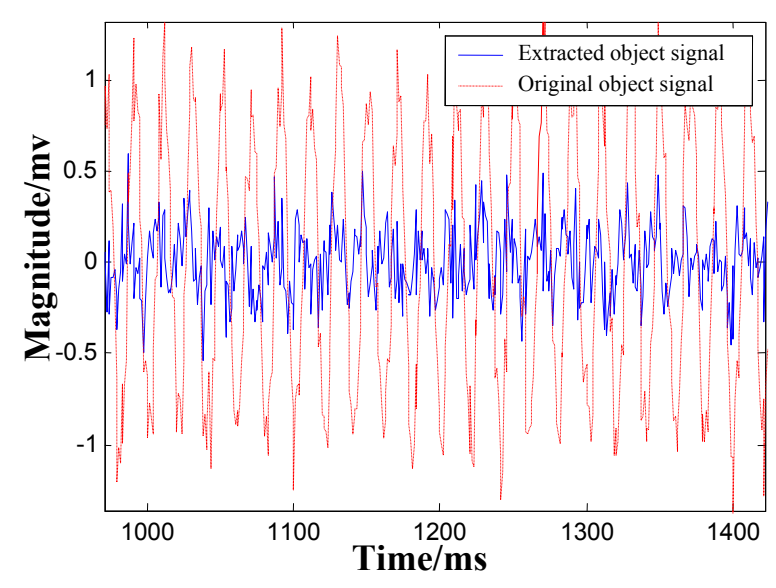

Fig.10. Signal after direct blast rejection (space angle of interference and signal is $1 \mathrm{deg}$ )

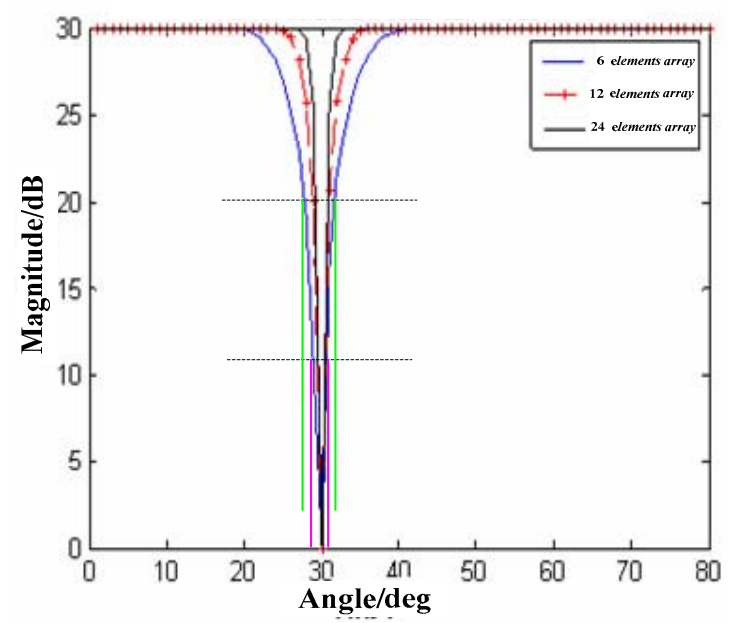

Fig.11. Performance comparison of direct blast rejection with different beamwidth

\section{Conclusion}

Direct blast is the strong interference in bistatic acoustic testing, the object scattering signal is impossible to be extracted from the strong direct blast interference. the algorithm of direct blast interference rejection based on vector sensor adaptive array processing fully take the advantage of adaptive filtering and vector senor array beamforming, which could reject the direct blast interference effectively with small array aperture. Simulation test results show that when the space angle of interference and signal greater than $5 \mathrm{deg}$, the object scattering signal can be effectively estimated with six elements of vector sensor array.

\section{Acknowledgement}

In this paper, the research was sponsored by Foundation of Science and Technology on Underwater Test and Control Laboratory (Project No. 9140C260201130C260).

\section{References}

[1] Stefano Coraluppi. Multistatic Sonar Localization[J]. IEEE Journal of Oceanic Engineering, Vol. 31(4), October, 2006. 964-974.

[2] Henry Cox, Fundamentals of bistatic active sonar[A]. Underwater acoustic data processing[C]. Klvwer academic publishers, 1989. 3-24.

[3] Michael Sandys-Wunsch and Mark G. Hazen. Multistatic Localization Error Due to Receiver Positioning Errors[J]. IEEE Journal of Oceanic Engineering, Vol. 27, NO. 2, April, 2002.

[4] Sun Da-jun, Zhou Ji-wu. A Study on Tank Experiment of Direct Blast Suppression in Bistatic Sonar[J]. IEEE, 2011.1-4.

[5] Gao Jie, Hou Wei-min. An Adaptive Algorithm of Direct Path Interference Suppression for Active Sonar Detection[J]. Technical Acoustics, 2008,537-540.

[6] Yao Yao, Zhang Min-Ming. "A Direct path Interference Suppression Algorithm of Bistatic testing Based on Adaptive Weighted Spatial Smoothing[C]. The 2011 Asia-Pacific Youth Conference of Youth Communication and Technology. 2011.28-31.

[7] Sun Gui-qing, Li Qi-hu. Acoustic vector sensor signal processing [J].ACTA ACUSTICA, Vol.29,NO.6,2004.491-498. 